\title{
European Hedgehogs (Erinaceus europaeus L.) as a Reservoir of Dermatophytes in Poland
}

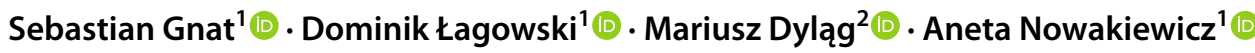

Received: 22 June 2021 / Accepted: 7 September 2021 / Published online: 18 September 2021

(c) The Author(s) 2021

\begin{abstract}
The European hedgehog (Erinaceus europaeus Linnaeus) frequently colonises areas located close to human life in cities, as these are more suitable nest sites offering an abundance of food and allowing avoidance of predators. However, urbanisation has a significant impact on the epidemiology of infectious diseases, including dermatophytoses, the primary source of which are wild animals. In this study, we determined the spectrum of dermatophytes isolated from the European hedgehog and assessed their susceptibility profile to antifungal drugs. Symptomatic and asymptomatic dermatophyte infections were observed in $7.7 \%$ and $8 \%$ of the 182 examined free-living hedgehogs, respectively. In the pool of the isolated dermatophyte strains, Trichophyton erinacei was dominant (29.9\%), followed by Trichophyton mentagrophytes (17.9\%), Trichophyton benhamiae (13.4\%), Nannizzia gypsea (11.9\%), Microsporum canis (10.4\%), Nannizzia nana (7.5\%), Paraphyton cookei (6.0\%), and Nannizzia fulva (3.0\%). Susceptibility tests revealed the highest activity of luliconazole and the lowest of activity fluconazole among the azole drugs applied. Although terbinafine generally exhibited high efficacy, two Trichophyton mentagrophytes isolates showed resistance to this drug $(\mathrm{MIC}=2 \mu \mathrm{g} / \mathrm{ml})$ resulting from missense mutations in the SQLE gene corresponding to the amino acid substitution Leu393Phe. Summarising, our study has also revealed that such wildlife animals as hedgehogs can be a reservoir of pathogenic human dermatophytes, including harmful strains resistant to commonly used antifungal drugs.
\end{abstract}

Keywords Erinaceus europaeus $\cdot$ Dermatophyte reservoir $\cdot$ Prevalence $\cdot$ Wildlife $\cdot$ Urban areas $\cdot$ Zoonoses

\section{Introduction}

In the last few years, superficial infections caused by filamentous fungi, especially dermatophytes, along with the concomitant increase in the number of difficult-to-treat cases have increasingly been recognised worldwide as a serious public health problem [1-3]. This imposes a high economic burden, as approximately $\$ 1.67$ billion is spent on the treatment of dermatophytosis each year [4]. The main etiological factors of superficial mycoses are dermatophytes, which are a cosmopolitan group encompassing more than 50 species classified within the genera Trichophyton, Microsporum,

Sebastian Gnat

sebastian.gnat@up.lublin.pl

1 Department of Veterinary Microbiology, Faculty of Veterinary Medicine, University of Life Sciences, Akademicka 12, 20-033 Lublin, Poland

2 Department of Mycology and Genetics, Faculty of Biological Sciences, University of Wroclaw, Wroclaw, Poland
Epidermophyton, Arthroderma, Nannizzia, Lophophyton, Guarromyces, and Paraphyton [5]. The sources of dermatophytes include the natural environment, i.e. soil (geophilic species), and transmission via direct or indirect contact with infected humans (antropophilic species) or animals (zoophilic species) as well as asymptomatic carriers [6-8].

The widely reported factors that predispose to human dermatophytosis include improper hygiene, occlusive footwear, socioeconomic conditions, profession, animal breeding, diabetes mellitus, age, genetics, skin maceration, and immunocompromised status [7, 9, 10]. Moreover, contact with domestic and wild animals should also be considered a risk factor $[11,12]$. The changing natural environment is favourable for these animals to colonise sites located close to human life in cities. It is commonly known that urbanisation has a significant impact on the epidemiology of infectious diseases, including dermatophytoses [13]. Many interventions, also nonprofessional in wildlife rescue, raise questions concerning the risk of transmission of zoophilic dermatophytes to volunteers. Additionally, the prevalence of 
symptomatic infections and the carriage of dermatophytes in wild animals have not yet been defined in detail.

Adaptation to urban and suburban areas is characteristic of the populations of the European hedgehog (Erinaceus europaeus Linnaeus, 1758), also known as the West European hedgehog or the common hedgehog $[14,15]$. In the $80 \mathrm{~s}$ and $90 \mathrm{~s}$ of the last century, a decline of the hedgehog population was described in western and central European regions, which was reflected in the inclusion of the species in Appendix III of the Bern Convention on the Conservation of European Wildlife and Natural Habitats [16]. The potential cause of this phenomenon may be the aftermath of the loss of their natural habitat and food sources after urbanisation, agriculture intensification, and the use of pesticides [17]. Other studies indicate that hedgehogs may prefer urban areas, as they offer more suitable nest sites and ensure a lower risk of predation from European badgers (Meles meles) [18]. Although hedgehogs residing in urban areas primarily become active after midnight and avoid foraging near roads, which is likely to reduce the dangers and disturbances caused by human activities such as vehicle and foot traffic and the disturbances from dogs, their contact with humans and pets is reported more frequently every year $[19,20]$. The major microbial infections associated with hedgehogs have bacterial aetiology, i.e. Salmonella spp. and Mycobacterium spp. [21, 22]. Furthermore, it is commonly known that European hedgehogs can also constitute an essential reservoir of dermatophytes, especially Trichophyton erinacei $[12,16]$. Nevertheless, the available information about the prevalence of dermatophytosis and carriage status in hedgehogs is based on a small dataset of free-ranging healthy hedgehogs or on a larger number of sick-injured hedgehogs in wildlife rescue centres. Hence, there seems to be a necessity to control the prevalence of dermatophytes in hedgehogs and to establish health precautions to avoid zoonotic infections. The latter may also apply to children who have contact with soil or pets encountering hedgehogs or their excrements, including keratinised tissue fragments, on the property of their owners.

The aim of this study was to determine the prevalence of dermatophytes on the body integuments of European hedgehogs in Poland. Comparative analyses were carried out over a 5-year period. In addition, species of dermatophytes were identified using conventional and molecular methods to indicate dermatophyte species associated with hedgehogs. Finally, antifungal drug susceptibility tests were performed due to the common phenomenon of antifungal drug resistance among strains of zoophilic dermatophytes [23-25], whose growing number is a potential risk for public health (Fig. 1).

\section{Materials and Methods}

\section{Material Sampling from Hedgehogs}

In total, 182 samples were collected from asymptomatic hedgehogs $(n=148)$ and those with dermatophytosis $(n=34)$ from six localities in different regions in Poland during the period from May to August in 2016-2020 (Table 1). Each location was a city with more than 100,000 inhabitants located in the eastern, central, and southwestern parts of Poland. All animals included in the study were free-living specimens in urban areas. The field identification of Erinaceus europaeus $\mathrm{L}$. was based on exterior characteristics, i.e. fur colouration and spine striping, presence or absence of a mask on the muzzle, and presence and shape of a white spot on the ventral side. Dermatophytosis was considered symptomatic when such skin lesions as spike loss, crusty skin, and erythema were diagnosed according to the criterion proposed by Bexton and Nelson [26]. Clinical examination was
Fig. 1 Free-living hedgehog (Erinaceus europaeus Linnaeus) during clinical examination in urbanised areas. Asymptomatic animal (A) and hedgehog with multiple lesions $(\mathbf{B})$
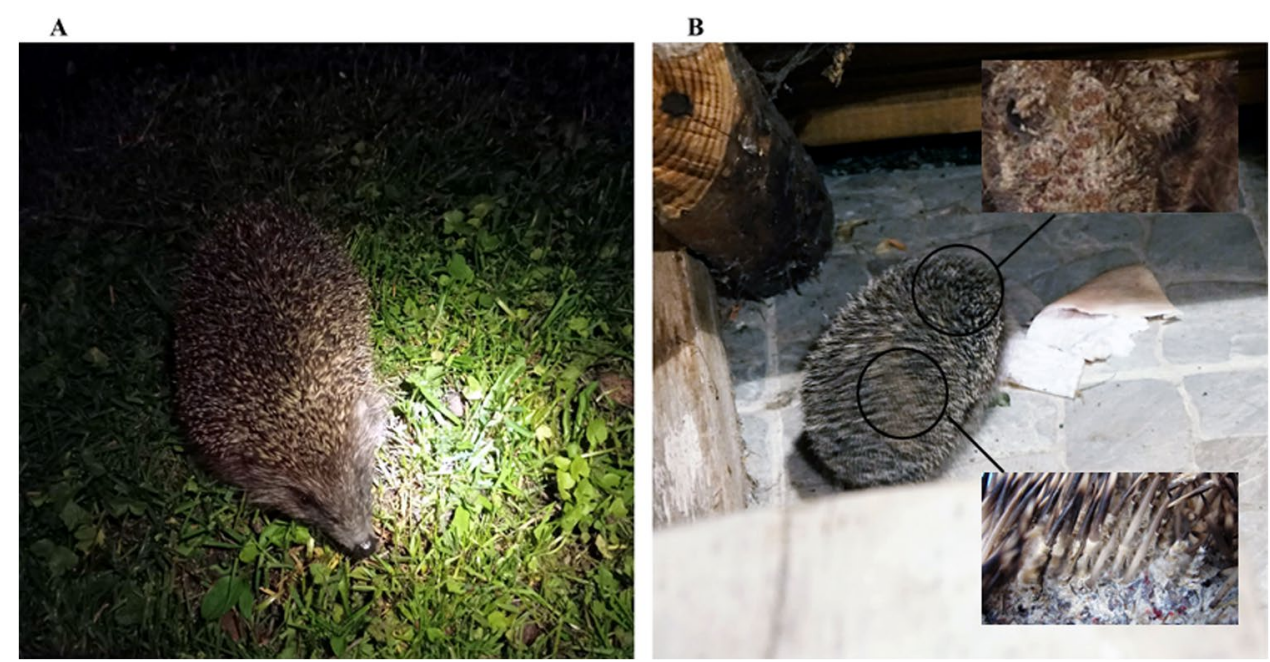
Table 1 Numerical data on the number of tested hedgehogs and the results obtained

\begin{tabular}{|c|c|c|c|c|}
\hline \multirow[t]{2}{*}{ Year } & \multicolumn{3}{|c|}{ Number of samples } & \multirow{2}{*}{$\begin{array}{l}\text { Number } \\
\text { of isolates } \\
(\%)\end{array}$} \\
\hline & Asymptomatic & Symptomatic & Total & \\
\hline 2016 & 28 & 6 & 34 & $13(38.2)$ \\
\hline 2017 & 35 & 5 & 40 & $10(25.6)$ \\
\hline 2018 & 26 & 15 & 41 & $19(46.3)$ \\
\hline 2019 & 21 & 3 & 24 & $9(39.1)$ \\
\hline 2020 & 38 & 5 & 43 & $14(32.5)$ \\
\hline Summarized & 148 & 34 & 182 & $67(36.8)$ \\
\hline
\end{tabular}

performed at the hedgehog encounter site without transferring the animal or any additional medicinal procedures in accordance with good veterinary practice standards. In the case of hedgehogs with symptomatic dermatophytosis, skin scrapings were collected by rubbing off a spike using a sterile scalpel and placed into a falcon tube. The asymptomatic animals were sampled by scrubbing the whole skin surface using the brush technique [27].

\section{Detection and Identification of Dermatophytes}

Mycological procedures, i.e. detection and species identification of the isolates, were performed with a few modifications as described previously by Gnat et al. [28]. The qPCR and microscopic methods were used to detect dermatophytes in the samples. Briefly, for the qPCR technique, DNA was extracted using a DNeasy Blood \& Tissue Kit (QIAGEN, Hilden, Germany) according to the manufacturer's instructions. The final positive result was obtained when the reactions with the pan-dermatophyte primers (primer F: AGC GCYCGCCGRAGGA, primer R: GATTCACGGAATTCT GCAATTCAC) were positive. The real-time PCR was performed in a final volume of $25 \mu \mathrm{l}$ containing $12.5 \mu \mathrm{l} 2 \times$ of
QuantiTect SYBR Green PCR Master Mix, $0.5 \mu \mathrm{l}(20 \mathrm{pmol})$ of both forward and reverse primers, $3 \mu \mathrm{l}$ of DNA, and $8.5 \mu \mathrm{l}$ of water. The cycling conditions of the reaction performed using Stratagene Mx3005P (Agilent Technologies, Santa Clara, USA) were as follows: primary denaturation for 3 min at $96{ }^{\circ} \mathrm{C}, 45$ denaturation cycles for $10 \mathrm{~s}$ at $96{ }^{\circ} \mathrm{C}$, annealing for $1 \mathrm{~min}$ at $65^{\circ} \mathrm{C}$, and elongation for $30 \mathrm{~s}$ at $72{ }^{\circ} \mathrm{C}$. The melting curve analysis was performed in the following conditions: $1 \mathrm{~min}$ at $94{ }^{\circ} \mathrm{C}, 1 \mathrm{~min}$ at $65^{\circ} \mathrm{C}$, and 1 min at $94{ }^{\circ} \mathrm{C}$. Direct examination of the skin scrapings was carried out using light and fluorescence microscopes after suspending the samples in clearing fluid comprising $10 \%$ $\mathrm{KOH}$ in a DMSO (dimethyl sulfoxide) solution. Microscopic preparations were then examined under a magnification of $400 \times($ Olympus BX51, Tokyo, Japan). (Fig. 2) For light microscopy, the preparations were examined after lactophenol blue and chlorazol black (Sigma-Aldrich, St. Louis, MO, USA) staining. Calcofluor white (Sigma-Aldrich, St. Louis, MO, USA) staining was used for fluorescence microscopy with 300-440 nm emission and ca. $355 \mathrm{~nm}$ excitation wavelengths.

Species identification was based on macro- and micromorphological examinations of the obtained cultures and ITS (internal transcribed spacer) region sequencing and analysis. (Fig. 3A and B) Cultures were inoculated onto Sabouraud glucose agar containing $0.005 \%$ chloramphenicol and $0.05 \%$ cycloheximide (BioMaxima, Lublin, Poland) and on dermatophyte test medium (BioMaxima, Lublin, Poland) at $30{ }^{\circ} \mathrm{C}$ for 21 days. Additionally, DNA was isolated from the axenic culture with the phenol-chloroform method described by Gnat et al. [29]. Molecular identification was performed by amplification of the internal transcribed spacer (ITS) region and nucleotide sequence analysis using the ITS1 (5'-TCCGTA GGTGAACCTGCGG-3') and ITS4 (5'-TCCTCCGCTTAT TGATATGC-3'; Genomed, Warsaw, Poland) primer pair
Fig. 2 Direct preparation from hedgehog skin scrapings stained with chlorazol black E magnified $400 \times($ Olympus BX51, Tokyo, Japan). Notes: $\mathbf{A}$, arthrospores in the skin scrap; $\mathbf{B}$, mycelium fragments indicated by arrows
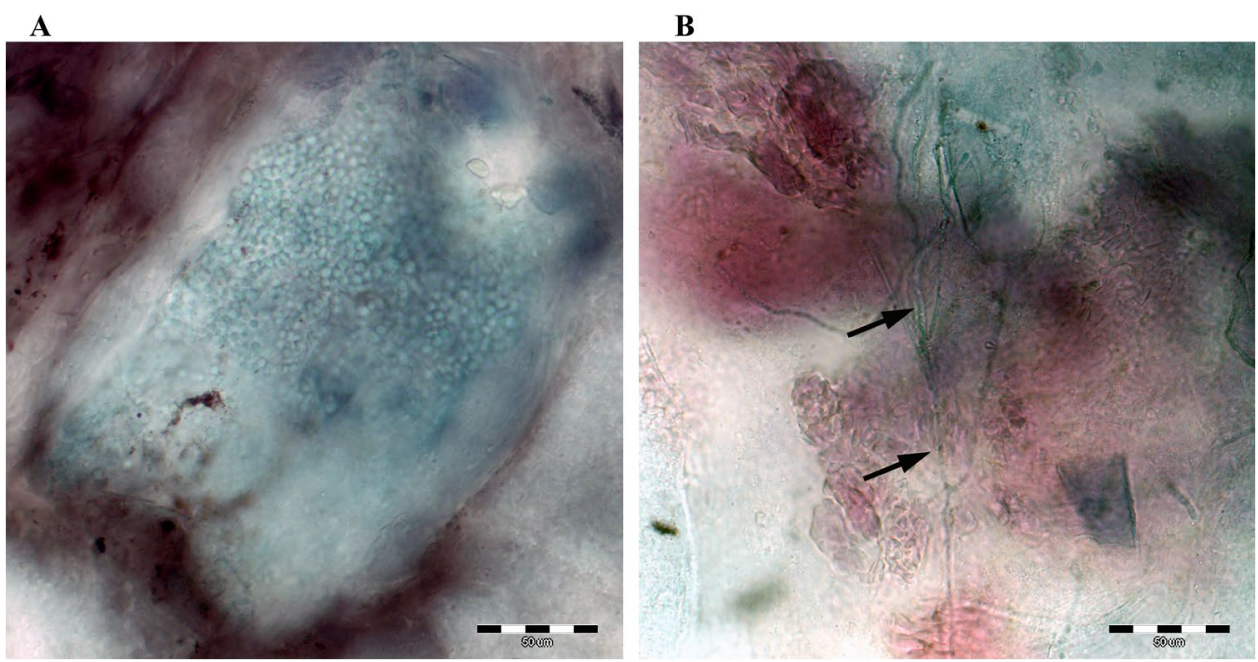

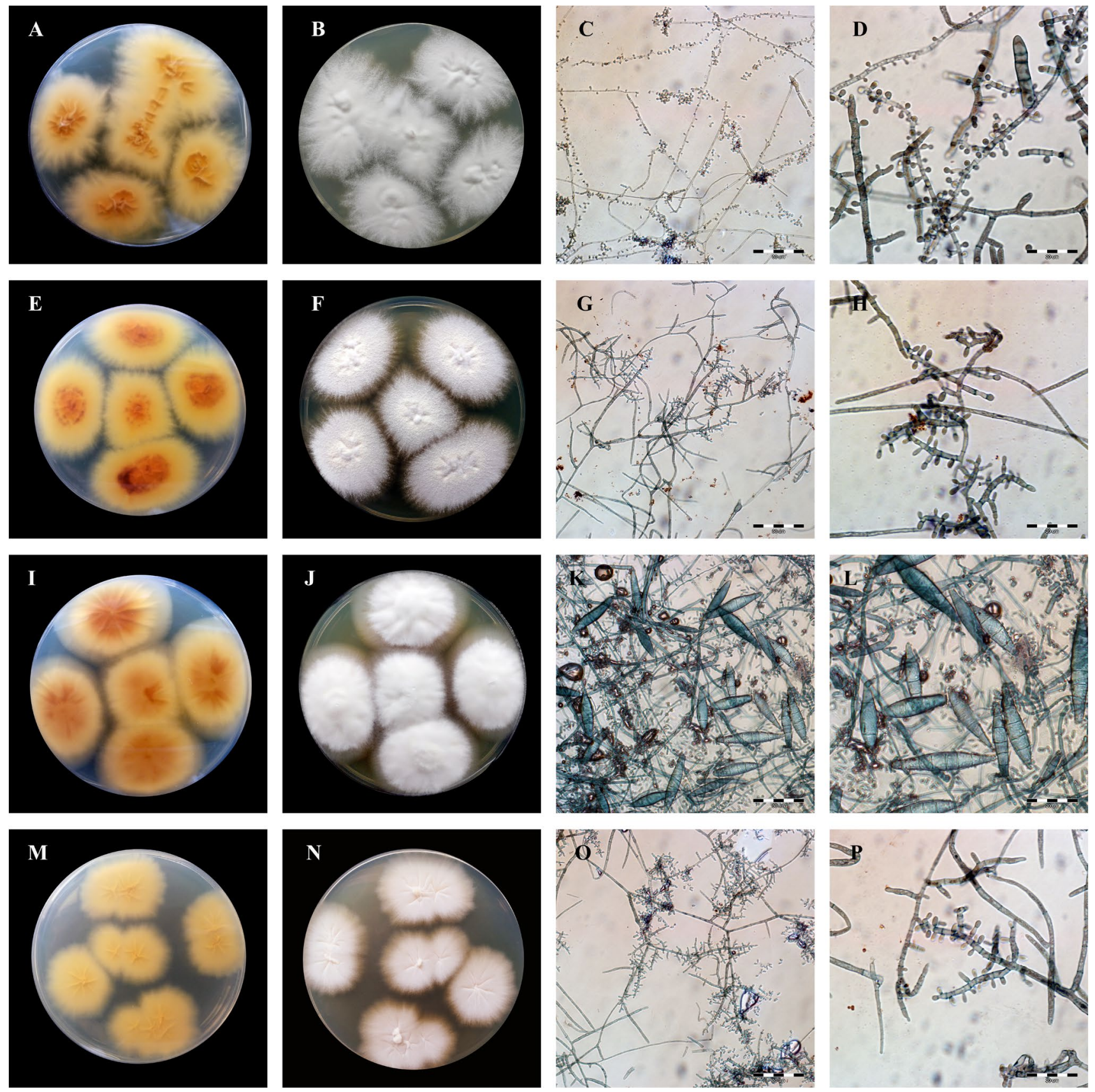

Fig. 3 Macro- and micromorphology of dermatophytes isolated from free-living hedgehogs. Notes: fungi cultured on Sabouraud medium at $28{ }^{\circ} \mathrm{C}$ for 21 days. A-D, Trichophyton mentagrophytes; E-H, Trichophyton benhamiae; I-L, Microsporum canis; M-P, Trichophyton erinacei. $\mathbf{A}, \mathbf{E}, \mathbf{I}$, and $\mathbf{M}$, obverse appearance; $\mathbf{B}, \mathbf{F}, \mathbf{J}$, and $\mathbf{N}$, reverse appearance; $\mathbf{C}, \mathbf{G}, \mathbf{K}$, and $\mathbf{O}$, micromorphology after staining with chlorazol black E at $400 \times$ magnification (Olympus BX51, Tokyo, Japan); D, H, L, and $\mathbf{P}$, micromorphology after staining with chlorazol black E at $1000 \times$ magnification (Olympus BX51, Tokyo,

[30]. The PCR reaction conditions were as follows: initial step at $95{ }^{\circ} \mathrm{C}$ for $3 \mathrm{~min}$ followed by 30 cycles at $95^{\circ} \mathrm{C}$ for $1 \mathrm{~min}, 50^{\circ} \mathrm{C}$ for $1 \mathrm{~min}$, and $72{ }^{\circ} \mathrm{C}$ for $1 \mathrm{~min}$, and then a

Japan). 3B Macro- and micromorphology of dermatophytes isolated from free-living hedgehogs continued. Notes: fungi cultured on Sabouraud medium at $28{ }^{\circ} \mathrm{C}$ for 21 days. A-D, Nannizzia gypsea; $\mathbf{E}-\mathbf{H}$, Paraphyton cookei; I-L, Nannizzia nana; M-P, Nannizzia fulva; A, $\mathbf{E}, \mathbf{I}$, and $\mathbf{M}$, obverse appearance; $\mathbf{B}, \mathbf{F}, \mathbf{J}$, and $\mathbf{N}$, reverse appearance; $\mathbf{C}, \mathbf{G}, \mathbf{K}$, and $\mathbf{O}$, micromorphology after staining with chlorazol black $\mathrm{E}$ at $400 \times$ magnification (Olympus BX51, Tokyo, Japan); D, H, L, and $\mathbf{P}$, micromorphology after staining with chlorazol black $\mathrm{E}$ at $1000 \times$ magnification (Olympus BX51, Tokyo, Japan)

final extension step at $72{ }^{\circ} \mathrm{C}$ for $10 \mathrm{~min}$. Electrophoretic separation of PCR products was carried out in $2 \%$ agarose gels. The ITS sequencing reaction was carried out using 

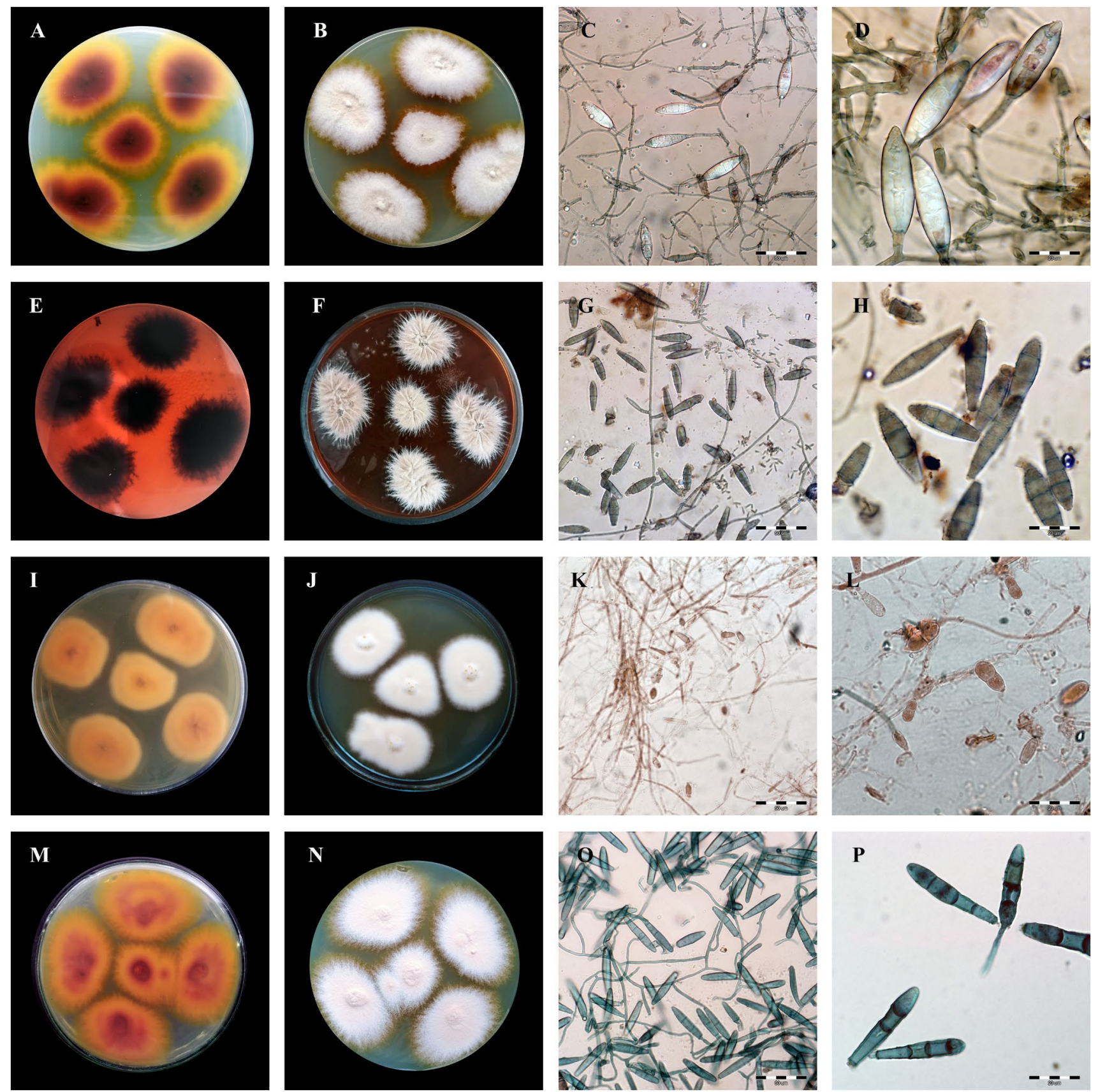

Fig. 3 (continued)

a BigDye Terminator Cycle Sequencing Kit (Life Technologies, Carlsbad, CA, USA). Two separate reactions were carried out using either the ITS 1 or ITS4 primer. The PCR product was purified using an ExTerminator kit (A\&A Biotechnology, Gdynia, Poland), and then the DNA sequence was read in a 3500 Genetic Analyser (Life Technologies, Carlsbad, CA, USA). All obtained nucleotide sequences were deposited in GenBank (Table 1).

\section{Susceptibility Testing to Antifungal Drugs}

In vitro susceptibility of isolated dermatophyte strains towards allylamine, polyene, imidazole, triazole, and pyridinone derivative drugs was determined according to Clinical and Laboratory Standards Institute (CLSI) document M38Ed3 [31]. Reagent-grade amphotericin B (AMB), ciclopirox (CPO), griseofulvin (GRE), itraconazole (ITR), ketoconazole (KTC), miconazole (MCZ), naftifine (NFT), 
terbinafine (TRB), and voriconazole (VRC) were obtained in the powder form. Drug stock solutions were prepared in dimethyl sulfoxide (DMSO) to reach the final DMSO concentration in the wells below $1 \%$. The drugs were analysed in two-fold serial dilutions in the range of $0.001-32 \mu \mathrm{g} /$ $\mathrm{ml}$. The dermatophyte isolates were cultured on potato dextrose agar (PDA; Difco) for 21 days, and suspensions comprising mostly conidia were prepared by gently scraping mature colonies into sterile physiological saline containing $0.002 \%$ Tween 80 . Homogeneous inoculum supernatants were collected, their optical density (OD) at $530 \mathrm{~nm}$ was adjusted spectrophotometrically to transmission ranging from 65 to $70 \%$, and the final density of the inoculum was from $1 \times 10^{3}$ to $3 \times 10^{3} \mathrm{CFU} / \mathrm{ml}$. The inocula were diluted 1:50 in RPMI 1640 medium and incubated with the indicated concentrations of the antifungals in 96-well plates at $30{ }^{\circ} \mathrm{C}$ for $72 \mathrm{~h}$. Minimum inhibitory concentrations (MICs) were read spectrophotometrically using a Varioskan LUX multimode microplate reader (Thermo Fisher Scientific) at $530 \mathrm{~nm}$ wavelength $(\lambda)$. The endpoint for the minimal inhibitory concentration (MIC) was the antifungal concentration at which prominent inhibition of growth, i.e. $\geq 80 \%$ of that of the control was observed. Trichophyton interdigitale ATCC4439 and T. rubrum ATCC4438 served as quality controls for every new series of susceptibility tests. All the tests were performed in triplicate and each tested compound used in this study was purchased from Sigma-Aldrich (MO, USA) if not stated otherwise. In addition, the selection of the terbinafine-resistant T. mentagrophytes isolates was based on fungal growth on Sabouraud glucose agar (SGA, BioMaxima, Lublin, Poland) containing $1 \mu \mathrm{g} / \mathrm{ml}$ of this substance, and the growth rate was compared to the control (SGA without drug) after 7,10 , and 14 days.

\section{Squalene Epoxidase (SQLE) Gene Analysis for Terbinafine-Resistant T. mentagrophytes}

Partial squalene epoxidase gene sequences for terbinafineresistant isolates were analysed as previously described by Yamada et al. [32] with a few modifications. In short, the SQLE gene amplification reaction was carried out with primer pairs TrSQLE-F1 (5'-ATGGTTGTAGAGGCTCCT CCC-3') and TrSQLE-R1 (5'-CTAGCTTTGAAGTTCGGC AAA-3') and DNA template with a concentration of $50 \mathrm{ng} /$ $\mu \mathrm{l}$. The PCR reaction conditions were as follows: 30 cycles consisting of denaturation $\left(1 \mathrm{~min}\right.$, at $\left.95^{\circ} \mathrm{C}\right)$, primer annealing $\left(30 \mathrm{~s}\right.$, at $\left.55^{\circ} \mathrm{C}\right)$, and elongation $\left(3 \mathrm{~min}\right.$, at $\left.72{ }^{\circ} \mathrm{C}\right)$. The PCR products were separated on $2 \%$ agarose gel stained with ethidium bromide and visualised in UV light. The SQLE gene sequencing reaction was carried out using a BigDye Terminator Cycle Sequencing Kit (Life Technologies, Carlsbad, CA, USA) and one of the primers TrSQLE-F1 or TrSQLE-R1 purified using an ExTerminator kit (A\&A Biotechnology,
Gdynia, Poland). Next, the DNA sequence was read in a 3500 Genetic Analyser purchased from Life Technologies (Carlsbad, CA, USA). The nucleotide and predicted amino acid sequences obtained in MEGA ver. 7.0 software (https://www. megasoftware.net/) of the SQLE gene in the case of all the T. mentagrophytes isolates were compared with the reference sequences available in the GenBank database.

\section{Results}

In total, 182 free-living hedgehogs were examined in this study. The veterinary analysis performed during the collection of the material showed 98 adult animals (53.9\%), 65 juveniles (35.7\%), and 19 hoglets (10.4\%). Moreover, the sex rate was not homogeneous for the three age groups, and 109 hedgehogs were male $(59.9 \%)$ and 73 animals were female $(40.1 \%)$. During the field examination of hedgehogs, the veterinarian observed fleas (Archaeopsylla erinacei) on 42 animals (23.1\%) and ticks (Ixodes sp.) on 21 animals $(11.5 \%)$ or both ectoparasites on 34 animals (18.7\%), with variable degrees of infestation. Thirty-one out of the 182 hedgehogs (17\%) had skin lesions diagnosed as cutaneous myiasis. Calliphoridae (Diptera) eggs and/or larvae were detected in these animals. Additionally, in 20 hedgehogs with cutaneous myiasis, there were 12 animals (6.6\%) with symptomatic dermatophytosis and 8 specimens (4.4\%) were asymptomatic carriers.

The qPCR technique facilitated the detection of the genetic material of dermatophytes in $100 \%$ of samples taken from the symptomatic hedgehogs (34/34). Moreover, this method used to analyse the material from the asymptomatic carrier animals revealed that $45.3 \%$ (67/148) of the samples contained dermatophyte genetic material (Table 2). In turn, the direct light and fluorescence microscopy examination of the material sampled from the skin lesions in the hedgehogs revealed the presence of arthrospores in all samples. During the diagnostics of the skin scrapings from the asymptomatic animals, fungal elements were shown by the direct examination with light and fluorescence microscopy in 33.8\% (50/148) and 37.8\% (56/148) of the analysed cases, respectively. In turn, dermatophyte cultures were obtained from $36.8 \%$ hedgehogs (67/182), with 20 samples positive to T. erinacei (29.9\%), 12 samples positive to T. mentagrophytes (17.9\%), 9 samples positive to $T$. benhamiae (13.4\%), and 26 samples positive to other species of dermatophytes $(41.8 \%)$. All dermatophyte strains cultured in this study are presented in Table 3. The macro- and micro-morphological characteristics of the isolates classified within individual species were specific and allowed initial identification (Fig. 1). The main diagnostic criteria to distinguish between these species were the colony diameter and appearance, the 
Table 2 Diagnostic effectiveness of qPCR, direct microscopy, and culture methods in relation to clinical material taken from hedgehogs

\begin{tabular}{|c|c|c|c|c|c|}
\hline \multirow[t]{3}{*}{ Type of infection } & \multicolumn{5}{|c|}{ Method [\% of positive results] } \\
\hline & \multirow[t]{2}{*}{$\mathrm{qPCR}$} & \multicolumn{2}{|l|}{ Direct analysis } & \multicolumn{2}{|l|}{ Cultures } \\
\hline & & $\begin{array}{l}\text { In light micros- } \\
\text { copy }\end{array}$ & $\begin{array}{l}\text { In fluorescence } \\
\text { microscopy }\end{array}$ & $\begin{array}{l}\text { Less than } 10 \\
\text { colonies }\end{array}$ & $\begin{array}{l}\text { More } \\
\text { than } 10 \\
\text { colonies }\end{array}$ \\
\hline Symptomatic & 100 & 100 & 100 & 64.7 & 35.3 \\
\hline Asymptomatic & 45.3 & 33.8 & 37.8 & 18.9 & 3.4 \\
\hline
\end{tabular}

presence of spiral hyphae, and species-specific macroconidia [33]. Furthermore, the same proportion of males $(38,56.7 \%)$ and females $(29,43.3 \%)$ were dermatophytepositive in the culture tests. Similar proportions were also observed in dermatophyte culture-positive hedgehogs classified by age: 24 out of 67 were adults (35.8\%), 22 were juveniles $(32.8 \%)$, and 21 were hoglets $(31.3 \%)$. Chi-square tests of independence showed no association between the detection of dermatophytes and the sex and age groups $(p>0.05)$. Additionally, 34 hedgehogs $(50.7 \%)$ had clinical lesions indicative of dermatophytosis. There were only 12 out of the 34 symptomatic hedgehogs with cultures yielding 10 or more colonies per plate $(35.3 \%)$, and no statistical association between the number of dermatophyte colonies and the presence of skin lesions was observed $\left(\chi^{2}, p>0.05\right)$. In turn, 20 of the symptomatic animals had wounds on the skin or were infested by Calliphoridae eggs and larvae (58.8\%). The statistical analysis revealed a significant relationship between these variables, i.e. animals with skin wounds were more likely to suffer from dermatophytosis $(\chi 2, p<0.05)$. The head of the hedgehogs was observed as the most frequent site of infection, as 21 animals presented skin lesions in this body area (61.8\%). Asymptomatic hedgehogs with isolated dermatophytes constituted 33 samples out of 148 subjects $(22.3 \%)$. Cultures with less than ten colonies per plate were obtained from 28 of these positive animals $(84.8 \%)$.

The $\mathrm{MIC}_{50}$ and $\mathrm{MIC}_{90}$ values of the thirteen antifungal drugs tested in the pool of 67 dermatophyte isolates obtained from the symptomatic and asymptomatic hedgehogs are summarised in Table 4. Luliconazole exhibited the lowest $\mathrm{MIC}_{50}$ and $\mathrm{MIC}_{90}$ values in comparison with the other drugs, regardless of the dermatophyte species. In turn, fluconazole was found to exert the weakest in vitro effect and had the highest $\mathrm{MIC}_{50}$ and $\mathrm{MIC}_{90}$ values. Although terbinafine exhibited high efficacy and low $\mathrm{MIC}_{50}$ values for all tested dermatophytes, two clinical isolates of $T$. mentagrophytes obtained from the symptomatic animals showed resistance to this substance ( $\mathrm{MIC}=2 \mu \mathrm{g} / \mathrm{ml}$ ). The verification test was performed on Sabouraud's medium supplemented with $1 \mu \mathrm{g}$ / $\mathrm{ml}$ of terbinafine to confirm the resistance of these isolates to the drug. The partial sequences of the SQLE gene obtained for these terbinafine-resistant $T$. mentagrophytes strains (GenBank accession numbers: MZ065195 and MZ065196) harboured missense mutations corresponding to the amino acid substitution Leu393Phe.

Table 3 Dermatophyte species isolated from hedgehogs with the GenBank accession numbers of the nucleotide sequences

\begin{tabular}{|c|c|c|c|c|}
\hline \multirow[t]{2}{*}{ Dermatophyte species } & \multirow[t]{2}{*}{ Type of infection } & \multicolumn{2}{|c|}{ Number of isolates } & \multirow{2}{*}{$\begin{array}{l}\text { GenBank accession numbers of ITS region and SQLE gene } \\
\text { nucleotide sequences }\end{array}$} \\
\hline & & No & Total $(\%)$ & \\
\hline \multirow[t]{2}{*}{ Trichophyton erinacei } & Symptomatic & 12 & $20(29.9)$ & MW755314-MW755325 \\
\hline & Asymptomatic & 8 & & MW755306-MW755313 \\
\hline \multirow[t]{2}{*}{ Trichophyton mentagrophytes } & Symptomatic & 8 & $12(17.9)$ & $\begin{array}{l}\text { MW759405-MW759412 } \\
\text { SQLE (2 strains): MZ065195-MZ065196 }\end{array}$ \\
\hline & Asymptomatic & 4 & & MW759878-MW759881 \\
\hline \multirow[t]{2}{*}{ Trichophyton benhamiae } & Symptomatic & 5 & $9(13.4)$ & MW759866-MW759870 \\
\hline & Asymptomatic & 4 & & MW759871-MW759874 \\
\hline \multirow[t]{2}{*}{ Microsporum canis } & Symptomatic & 4 & $7(10.4)$ & MW759862-MW759865 \\
\hline & Asymptomatic & 3 & & MW759875-MW759877 \\
\hline \multirow[t]{2}{*}{ Nannizzia gypsea } & Symptomatic & 3 & $8(11.9)$ & MW759402-MW759404 \\
\hline & Asymptomatic & 5 & & MW759413-MW759417 \\
\hline Nannizzia nana & Asymptomatic & 5 & $5(7.5)$ & MW755440-MW755444 \\
\hline Nannizzia fulva & Symptomatic & 2 & $2(3.0)$ & MZ052087-MZ052088 \\
\hline Paraphyton cookei & Asymptomatic & 4 & $4(6.0)$ & MW755326-MW755329 \\
\hline
\end{tabular}




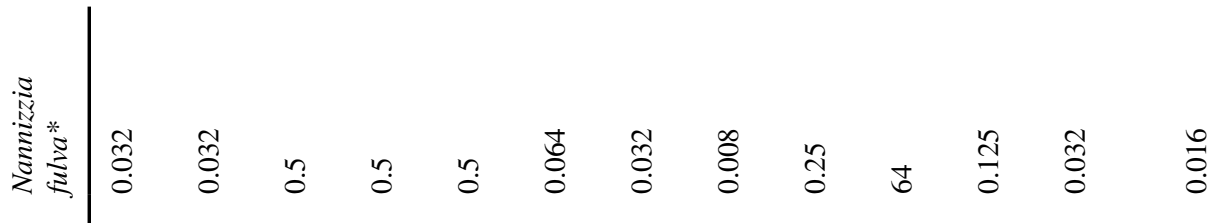

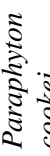

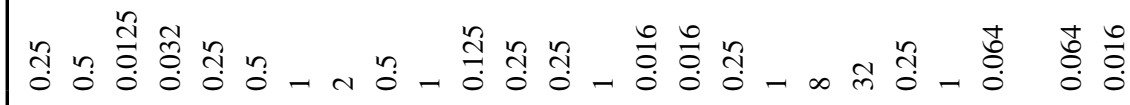

$\stackrel{\circ}{\circ}$

踏

害

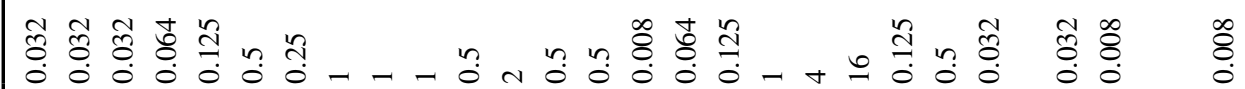

| 空

:

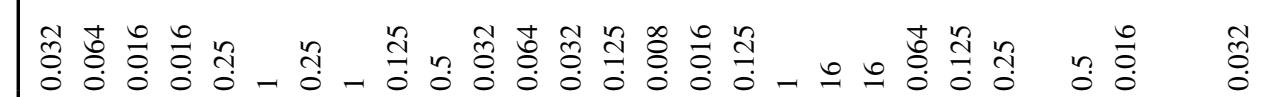

$\sqrt{2}$

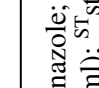

ठ․ㅇ

$\Leftrightarrow \pi$

退焉

要

정

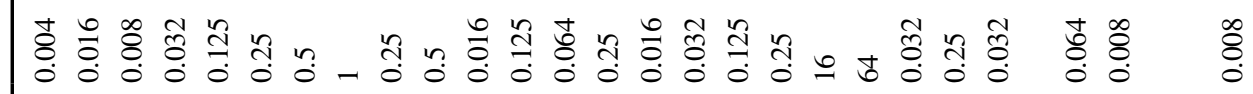

ชิ

萡

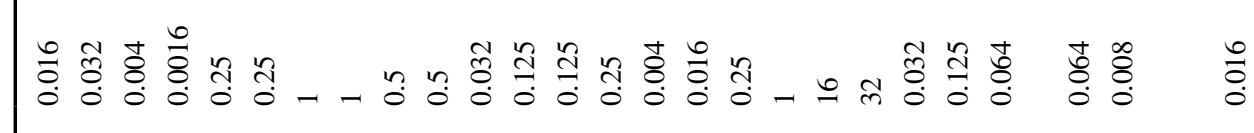

远

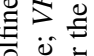

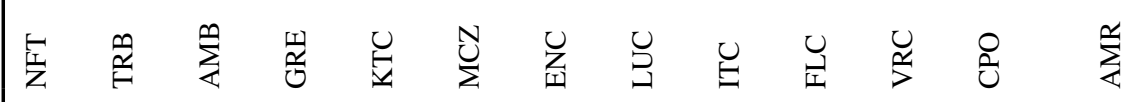




\section{Discussion}

The European hedgehog (Erinaceus europaeus Linnaeus, 1758 ) is a nocturnal insectivorous mammal with a wide distribution throughout Europe [22, 34]. The hedgehog population has increased in urban areas, especially in cities $[12,17]$. Although hedgehogs spend most of the daylight hours sleeping and emerge at night to forage, interactions between hedgehogs and other animals and humans are on the rise nowadays $[22,35]$. This is confirmed by reports on contact urticaria in some handlers and a number of infectious zoonotic diseases associated with hedgehogs $[21,36,37]$. Several reports also demonstrate the ability of hedgehogs to transmit dermatophytes to humans, resulting in zoonotic disease [38, 39] [40]. Hence, monitoring of hedgehog populations in urban areas has become of utmost importance if we consider the role of wild animals as carriers of dermatophytes and related fungi.

In our study, the total rate of culture-positive samples from hedgehogs was $36.8 \%$. This percentage is consistent with earlier results, although these studies were conducted in the 1960s. In 1964, Smith and Marples [41] described a higher prevalence of dermatophyte isolates $(44.7 \%)$ within urban wild populations in New Zealand. In turn, in studies conducted in 1969 in Great Britain, Morris and English [42] found a lower percentage of dermatophytoses in hedgehogs (20-25\%). In a more recent study conducted in wildlife rehabilitation centres in France, Le Barzic et al. [12] revealed a $25.4 \%$ rate of dermatophyte-positive samples from hedgehogs. Moreover, the same study showed $79.2 \%$ of positive cultures coming from asymptomatic hedgehogs [12]. This percentage is much higher than that obtained in our study. The asymptomatic dermatophyte-positive hedgehogs constituted only $22.3 \%$, and cultures with less than ten colonies per plate were obtained in $84.8 \%$ of these samples. The differences between these results and those obtained in our study may be associated with the method of collecting samples. The wild animal rescue centre where skin scrapings were collected in the French studies houses sick, injured, or malnourished animals, in health status that may predispose them to dermatophyte infections and asymptomatic carriage. Therefore, all veterinary activities with wild animals should be performed using protective gloves and continuous environmental disinfection to avoid contagion [21, 22]. In the natural environment, the risk of transmission of infection from hedgehogs is lower, and more attention should be paid to soil contamination, as it can indirectly pose a risk of infection to humans and other animals, including pets. It is commonly known that some zoophilic dermatophytes also have soil reservoirs [24].

The isolation and identification of the typically geophilic dermatophyte Nannizzia gypsea, as well as a species of dual zoophilic and geophilic nature, i.e. Nannizzia nana, from free-living hedgehogs confirms that the reservoirs of dermatophytes can change and the soil is a favourable environment for these pathogens. It should be noted that Nannizzia fulva was isolated for the first time from hedgehogs in our study. This geophilic dermatophyte species was detected in a variety of soil sources, especially shady and wet places rich in organic substances, e.g. gardens, public grassland, sludge, and locations full of animal keratin materials in Iran [43, 44], Tunisia [45], India [46], Saudi Arabia [47], USA [48], and Brazil [49]. Additionally, $N$. fulva was more often isolated from animal habitats in parks and city gardens than N. gypsea, which is typically related to soils in home range areas [50]. Hence, hedgehogs found in urban regions may be infected. The presence of geophilic dermatophytes $N$. gypsea in asymptomatic carrier status animals was reported as well. These results are consistent with those published by Le Barzic et al. [12] based on research carried out in France at a wildlife rescue centre. This may indicate that hedgehogs are an important carrier of geophilic dermatophytes, regardless of the geographic region. Nonetheless, the hedgehog-specific fungus $T$. erinacei was the most frequent dermatophyte species observed (29.9\%) in both symptomatic and asymptomatic animals. The host range of this species seems to be narrow and identification thereof in other animals and in human cases of infection is rare compared to that in hedgehogs [51-54]. Contrarily, T. mentagrophytes found in $17.9 \%$ of cases in hedgehogs with dermatophytosis and asymptomatic carriers have been reported from a broad but poorly known spectrum of domestic animals and human zoophilic origin mycoses $[1,2,55,56]$. This species of dermatophyte has already been isolated from hedgehogs by Le Barzic et al. [12] in a monitoring study conducted in wildlife rehabilitation centres. Additionally, these authors indicate that T. mentagrophytes morphology is relatively similar to that of T. erinacei, which may cause confusion in identification analyses, especially samples from wild animals. In part, this is related to the extensive taxonomic rearrangements in the dermatophyte group since 2017 [5, 57]. Therefore, identification by molecular techniques should always be performed, as some isolates do not develop characteristic features to ensure the credibility of the morphological examination alone.

In the context of the isolation of T. mentagrophytes from hedgehogs, the detection of terbinafine-resistant strains is clinically important. Allylamine resistance has already been reported in Asian and European countries [2, 3, 32, 58-60]. Moreover, the prevalence of terbinafine-resistant clinical isolates of T. mentagrophytes ranged from less than 1 in Switzerland [32] to more than 70\% in India [61]. Current scientific reports do not clearly explain the cause of 
this phenomenon. Terbinafine resistance can be selected after treatment in the patient or can be innate [2, 62]. Our results are not able to indicate clearly the mechanism that might exert an effect on resistance in free-living hedgehogs. Nevertheless, in two cases, in vitro resistance to terbinafine ( $\mathrm{MIC}=2 \mu \mathrm{g} / \mathrm{ml}$ ) was demonstrated for T. mentagrophytes isolates obtained from animals with dermatophytosis. In addition, the Leu393Phe amino acid substitution in the squalene epoxidase protein was identified as responsible for the resistance. This substitution, together with Phe397Leu, was reported in earlier studies conducted on terbinafineresistant Trichophyton spp. isolates [2, 62-65]. Furthermore, Gln408Leu, Leu393Ser, Leu398Phe, Phe402Leu, and other less common substitutions were also correlated with high MIC values of terbinafine in dermatophytes and yeast-like fungi $[58,66]$. Many researchers report that terbinafine resistance is also geographically limited and regional predispositions to differential sensitivity to antifungal drugs are noted $[2,67]$. Based on the literature, it can be concluded that the greatest prevalence of resistant dermatophytes occurs in Asian countries, especially India. Nevertheless, European countries, including Poland [1, 2], Finland [59], Denmark [68], Switzerland [58], and Russia [60], are not free from dermatophyte isolates that are resistant to conventional treatment. Our study shows that hedgehogs may be a poorly studied reservoir of these strains. Moreover, the infectious agent of dermatophytoses in the free-living hedgehogs described here may have originated also from the soil, i.e. a non-animal environment that can be an additional reservoir of T. mentagrophytes. Therefore, the detection of drug-resistant strains, animal species affected, and disease state and the indication of their geographical range is an important task for medical mycologists.

A strikingly high incidence of zoonotic $T$. benhamiae infections is currently reported in various European countries [69]. The prevalence of this pathogen isolated from guinea pig breeds and pet shops reaches up to 90\% [70]. However, this dermatophyte has been also reported in dogs, rabbits, cats, North American porcupines, alpacas, foxes, and various small rodents [27, 71]. So far, T. benhamiae has not been reported in hedgehogs, either free-living or kept as pets, or any other wild animals. Probably due to the presence of this species of dermatophyte in rodents, whose feeding niches may be similar to those of urban hedgehogs, mechanical transmissions are possible. Thus, it is possible that the host range for T. benhamiae is wide, and subsequent monitoring studies will reveal new animal reservoirs. Moreover, the isolation of $M$. canis from urban hedgehogs seems interesting as well. This dermatophyte is usually correlated with a domestic environment and is associated with dogs and cats [72]. Nonetheless, in the last year, M. canis was isolated from dermatological lesions of Eastern cottontail (Sylvilagus floridanus), which is a synanthropic animal easily observed near urban centres with high population density. The fungus was also isolated from hair and soil samples collected in front of burrow entrances of alpine marmot (Marmota marmota) [73, 74]. This may indicate potential $M$. canis reservoirs in wild animals, especially those that forage in urban areas.

Recently, the literature has presented a suggestion that the molecular approach for the identification of dermatophytes in samples is trustworthy, rapid, and significantly more reliable than conventional diagnostic methods [28]. The real-time PCR techniques are increasingly frequently used in the detection of dermatophytes due to their high specificity and sensitivity, even in cases with negative cultures $[1,27,28]$. Our studies have proved that the use of the real-time PCR technique with pan-dermatophyte primers detects the presence of dermatophytes in the sample with a $35.3 \%(100 \%$ vs. $64.7 \%)$ and $26.4 \%(45.3 \%$ vs. $18.9 \%$ ) higher efficiency than cultures in the analysis of symptomatic and asymptomatic hedgehogs, respectively. Nevertheless, talking about the status of a carrier, it is necessary to indicate the presence of living infectious elements of dermatophytes [1,24]. These can only be demonstrated using conventional methods with obtaining cultures [28]. Therefore, the molecular methods for identifying dermatophytes cannot be used alone, without reference to obtaining culture by conventional methods.

In conclusion, the high prevalence of symptomatic infections and asymptomatic carriers in free-living hedgehogs detected in this study stresses the risk of dermatophyte dissemination in the natural environment and indicates possible zoonotic transmissions. The identification of geophilic dermatophyte species may also indicate the currently changing ecological niches of these fungi. Moreover, we have revealed that in vitro terbinafine resistance may emerge with the analysed mutations in the squalene epoxidase gene in wildlife animals. Finally, further research is needed to elucidate whether the asymptomatic carriage is related only to mechanical transport of dermatophytes or infection with low-virulence isolates as well.

Author Contribution S.G.: conceptualization, methodology, investigation, formal analysis, writing the original draft. D.Ł.: methodology, investigation, formal analysis, writing, reviewing, and editing. M.D.: formal analysis, writing, reviewing, and editing. A.N.: formal analysis.

Data Availability The datasets generated during the current study are available from the corresponding author on reasonable request.

\section{Declarations}

Ethics Approval The study was performed in accordance with the ethical standards consistent with good veterinary practices.

Conflict of Interest The authors declare no competing interests. 
Open Access This article is licensed under a Creative Commons Attribution 4.0 International License, which permits use, sharing, adaptation, distribution and reproduction in any medium or format, as long as you give appropriate credit to the original author(s) and the source, provide a link to the Creative Commons licence, and indicate if changes were made. The images or other third party material in this article are included in the article's Creative Commons licence, unless indicated otherwise in a credit line to the material. If material is not included in the article's Creative Commons licence and your intended use is not permitted by statutory regulation or exceeds the permitted use, you will need to obtain permission directly from the copyright holder. To view a copy of this licence, visit http://creativecommons.org/licenses/by/4.0/.

\section{References}

1. Gnat S, Łagowski D, Nowakiewicz A et al (2020) Population differentiation, antifungal susceptibility, and host range of Trichophyton mentagrophytes isolates causing recalcitrant infections in humans and animals. Eur J Clin Microbiol Infect Dis 39:20992113. https://doi.org/10.1007/s10096-020-03952-2

2. Łagowski D, Gnat S, Nowakiewicz A et al (2020) Intrinsic resistance to terbinafine among human and animal isolates of Trichophyton mentagrophytes related to amino acid substitution in the squalene epoxidase. Infection 48:889-897. https://doi.org/10. 1007/s15010-020-01498-1

3. Hiruma J, Kitagawa H, Noguchi H et al (2019) Terbinafineresistant strain of Trichophyton interdigitale strain isolated from a tinea pedis patient. J Dermatol 46:351-353. https://doi.org/10. 1111/1346-8138.14809

4. Gnat S, Łagowski D, Nowakiewicz A, Dyląg M (2021) A global view on fungal infections in humans and animals: opportunistic infections and microsporidioses. J Appl Microbiol n/a:jam.15032. https://doi.org/10.1111/jam.15032

5. de Hoog GS, Dukik K, Monod M et al (2017) Toward a novel multilocus phylogenetic taxonomy for the dermatophytes. Mycopathologia 182:5-31. https://doi.org/10.1007/s11046-016-0073-9

6. Łagowski D, Gnat S, Nowakiewicz A, et al (2019) The prevalence of symptomatic dermatophytoses in dogs and cats and the pathomechanism of dermatophyte infections. Postępy Mikrobiol - Adv Microbiol 58:165-176. https://doi.org/10.21307/pm-2019.58.2. 165

7. Gnat S, Nowakiewicz A, Łagowski D, Zięba P (2019) Host- and pathogen-dependent susceptibility and predisposition to dermatophytosis. J Med Microbiol 68:823-836. https://doi.org/10.1099/ jmm.0.000982

8. Dworecka-Kaszak B, Dąbrowska I (2017) Dermatofity - Nowa taksonomia i współczesne metody różnicowania Przegląd aktualnego stanu wiedzy o mechanizmach patogenezy i interakcjach patogen-gospodarz. Med Weter 73:613-617. https://doi.org/10. 21521/mw.5791

9. Gnat S, Łagowski D, Nowakiewicz A (2021) Genetic predisposition and its heredity in the context of increased prevalence of dermatophytoses. Mycopathologia. https://doi.org/10.1007/ s11046-021-00529-1

10. Salakshna N, Bunyaratavej S, Matthapan L et al (2018) A cohort study of risk factors, clinical presentations, and outcomes for dermatophyte, nondermatophyte, and mixed toenail infections. J Am Acad Dermatol 79:1145-1146. https://doi.org/10.1016/j.jaad. 2018.05.041

11. Albano APN, da Silva NP, Meirelles Leite AT et al (2013) Isolation of dermatophytes in wild felids from screening centers. Brazilian J Microbiol 44:171-174. https://doi.org/10.1590/S151783822013000100025
12. Le Barzic C, Cmokova A, Denaes C et al (2021) Detection and control of dermatophytosis in wild European hedgehogs (Erinaceus europaeus) admitted to a French wildlife rehabilitation centre. J Fungi 7:1-13. https://doi.org/10.3390/jof7020074

13. Hassell JM, Begon M, Ward MJ, Fèvre EM (2017) Urbanization and disease emergence: dynamics at the wildlife-livestock-human interface. Trends Ecol Evol 32:55-67. https://doi.org/10.1016/j. tree.2016.09.012

14. Rasmussen SL, Berg TB, Dabelsteen T, Jones OR (2019) The ecology of suburban juvenile European hedgehogs (Erinaceus europaeus) in Denmark. Ecol Evol 9:13174-13187. https://doi. org/10.1002/ece 3.5764

15. Williams BM, Baker PJ, Thomas E et al (2018) Reduced occupancy of hedgehogs (Erinaceus europaeus) in rural England and Wales: The influence of habitat and an asymmetric intra-guild predator. Sci Rep 8:12156. https://doi.org/10.1038/ s41598-018-30130-4

16. Contet-Audonneau N, Saboureau M, Percebois G (1991) Trichophyton erinacei chez le hérisson. J Mycol Med 1:29-32

17. Hubert P, Julliard R, Biagianti S, Poulle ML (2011) Ecological factors driving the higher hedgehog (Erinaceus europeaus) density in an urban area compared to the adjacent rural area. Landsc Urban Plan 103:34-43. https://doi.org/10.1016/j.landu rbplan.2011.05.010

18. Pettett CE, Moorhouse TP, Johnson PJ, Macdonald DW (2017) Factors affecting hedgehog (Erinaceus europaeus) attraction to rural villages in arable landscapes. Eur J Wildl Res 63:54. https://doi.org/10.1007/s10344-017-1113-6

19. Dowding CV, Harris S, Poulton S, Baker PJ (2010) Nocturnal ranging behaviour of urban hedgehogs, Erinaceus europaeus, in relation to risk and reward. Anim Behav 80:13-21. https:// doi.org/10.1016/j.anbehav.2010.04.007

20. Amory T, Committee BS (2008) Mortality factors affecting wild hedgehogs : a study of records from wildlife rescue centres. Lutra 42:7-9

21. Rosen T, Jablon J (2003) Infectious threats from exotic pets: dermatological implications. Dermatol Clin 21:229-236. https://doi.org/10.1016/S0733-8635(02)00090-6

22. Riley PY, Chomel BB (2005) Hedgehog zoonoses. Emerg Infect Dis 11:1-5. https://doi.org/10.3201/eid1101.040752

23. Gnat S, Łagowski D, Nowakiewicz A et al (2021) Complementary effect of mechanism of multidrug resistance in Trichophyton mentagrophytes isolated from human dermatophytoses of animal origin. Mycoses 64:537-549. https://doi.org/10.1111/ myc. 13242

24. Gnat S, Łagowski D, Nowakiewicz A, Dylagg M (2020) Unusual dermatomycoses caused by Nannizzia nana: the geophilic origin of human infections. Infection 48:429-434. https://doi.org/10. 1007/s15010-020-01416-5

25. Hiruma J, Noguchi H, Hase M et al (2021) Epidemiological study of terbinafine-resistant dermatophytes isolated from Japanese patients. J Dermatol 48:564-567. https://doi.org/10.1111/13468138.15745

26. Bexton S, Nelson H (2016) Comparison of two systemic antifungal agents, itraconazole and terbinafine, for the treatment of dermatophytosis in European hedgehogs (Erinaceus europaeus). Vet Dermatol 27:500-e133. https://doi.org/10.1111/vde.12378

27. Gnat S, Łagowski D, Nowakiewicz A et al (2020) Identification of emerging trends in the prevalence of dermatophytoses in alpacas (Vicugna pacos) farmed in Poland. Transbound Emerg Dis 67:2702-2712. https://doi.org/10.1111/tbed.13624

28. Gnat S, Łagowski D, Nowakiewicz A et al (2021) Detection and identification of dermatophytes based on currently available methods - a comparative study. J Appl Microbiol 130:278-291. https:// doi.org/10.1111/jam.14778 
29. Gnat S, Nowakiewicz A, Ziółkowska G et al (2017) Evaluation of growth conditions and DNA extraction techniques used in the molecular analysis of dermatophytes. J Appl Microbiol 122:13681379. https://doi.org/10.1111/jam.13427

30. White, Bruns T, Lee S, Taylor J (2007) White, T. J., T. D. Bruns, S. B. Lee, and J. W. Taylor. Amplification and direct sequencing of fungal ribosomal RNA genes for phylogenetics. pp 315-322

31. Clinical Laboratory Standards Institute (2017) Reference method for broth dilution antifungal susceptibility testing of yeast, 3rd ed. Clinical and Laboratory Standards Institute, Wayne,PA

32. Yamada T, Maeda M, Alshahni MM, et al (2017) Terbinafine resistance of Trichophyton clinical isolates caused by specific point mutations in the squalene epoxidase gene. Antimicrob Agents Chemother 61: https://doi.org/10.1128/AAC.00115-17

33. Elgart ML (1997) Atlas of clinical fungi. J Am Acad Dermatol 37:291. https://doi.org/10.1016/S0190-9622(97)80153-3

34. Szalay FS (2000) The Atlas of European Mammals . A. J. Mitchell-Jones , G. Amori , W. Bogdanowicz, B. Kryštufek, P. J. H. Reijnders, F. Spitzenberger, M. Stubbe , J. B. M. Thissen, V. Vohralik, J. Zima . Q Rev Biol 75:472-472. https://doi.org/10. $1086 / 393687$

35. Hoefer HL (1994) Hedgehogs. Vet Clin North Am - Small Anim Pract 24:113-120. https://doi.org/10.1016/S0195-5616(94) 50006-8

36. Fairley JA, Suchniak J, Paller AS (1999) Hedgehog hives. Arch Dermatol 135:561-563. https://doi.org/10.1001/archderm.135.5. 561

37. Maertens J, Vrebos M, Boogaerts M (2001) Assessing risk factors for systemic fungal infections. Eur J Cancer Care (Engl) 10:56-62. https://doi.org/10.1046/j.1365-2354.2001.00241.x

38. Philpot CM, Bowen RG (1992) Hazards from hedgehogs: two case reports with a survey of the epidemiology of hedgehog ringworm. Clin Exp Dermatol 17:156-158. https://doi.org/10.1111/j.13652230.1992.tb00193.x

39. Rosen T, Houston T (2000) Hazardous hedgehogs. South Med J 93:936-938. https://doi.org/10.1097/00007611-200093090-00023

40. Marples MJ, Smith JMB (1960) The hedgehog as a source of human ringworm. Nature 188:867-868. https://doi.org/10.1038/ $188867 \mathrm{~b} 0$

41. Smith JMB, Marples MJ (1964) Trichophyton mentagrophytes var. erinacei. Med Mycol 3:1-10. https://doi.org/10.1080/00362 176485190021

42. English MP, Morris P (1969) Trichophyton mentagrophytes var. erinacei in hedgehog nests. Med Mycol 7:118-121. https://doi. org/10.1080/00362177085190211

43. Rezaei-Matehkolaei A, Jahangiri A, Mahmoudabadi AZ et al (2017) Morpho-molecular characterization of soil inhabitant dermatophytes from Ahvaz, southwest of Iran, a high occurrence of Microsporum fulvum. Mycopathologia 182:691-699. https://doi. org/10.1007/s11046-017-0116-x

44. Dehghan P, Yousefi Jalali S, Chadeganipour M (2019) Frequency distribution of keratinophilic dermatophyte fungi from the soil of different zones in Isfahan using morphological and molecular methods. Adv Biomed Res 8:38. https://doi.org/10.4103/abr.abr_ 31_19

45. Anane S, Al-Yasiri MHY, Normand AC, Ranque S (2015) Distribution of keratinophilic fungi in soil across Tunisia: a descriptive study and review of the literature. Mycopathologia 180:61-68. https://doi.org/10.1007/s11046-015-9870-9

46. Balajee SAM, Ranganathan S, Menon T (1997) Soil dermatophytes in Madras, India, in relation to human ringworm. Mycoses 40:317-320. https://doi.org/10.1111/j.1439-0507.1997.tb00239.x

47. Rizwana H, Al Hazzani AA, Siddiqui I (2012) Prevalence of dermatophytes and other keratinophilic fungi from soils of public parks and playgrounds of Riyadh, Saudi Arabia. J Anim Plant Sci 22:948-953
48. Giugnani HC, Venketesan K (2020) Dermatophytes and other keratinophiic fungi in soils of Anguilla, British West Indies (BWI). Microbiol Res J Int 1-6. https://doi.org/10.9734/mrji/ 2020/v30i730233

49. Da Silva Pontes ZBV, Oliveira AC (2008) Dermatophytes from urban soils in João Pessoa, Paraíba, Brazil. Rev Argent Microbiol 40:161-163

50. Taghipour S, Abastabar M, Piri F, et al (2021) Diversity of geophilic dermatophytes species in the soils of Iran; the significant preponderance of Nannizzia fulva. J Fungi 7: https://doi.org/10. 3390/jof7050345

51. Hubka V, Peano A, Cmokova A, Guillot J (2018) Common and emerging dermatophytoses in animals: well-known and new threats. In: Seyedmousavi S, de Hoog GS, Guillot J, Verweij PE (eds) Emerging and epizootic fungal infections in animals. Springer International Publishing, Cham, pp 31-79

52. Phaitoonwattanakij S, Leeyaphan C, Bunyaratavej S, Chinhiran K (2019) Trichophyton erinacei onychomycosis: rhe first to evidence a proximal subungual onychomycosis pattern. Case Rep Dermatol 11:198-203. https://doi.org/10.1159/000501424

53. Rivaya B, Fernández-Rivas G, Cabañes FJ et al (2020) Trichophyton erinacei: an emergent pathogen of pediatric dermatophytosis. Rev Iberoam Micol 37:94-96. https://doi.org/10.1016/j.riam. 2020.06.001

54. Hsieh CW, Sun PL, Wu YH (2010) Trichophyton erinacei infection from a hedgehog: a case report from Taiwan. Mycopathologia 170:417-421. https://doi.org/10.1007/s11046-010-9333-2

55. Gnat S, Nowakiewicz A, Lagowski D et al (2019) Multiple-strain Trichophyton mentagrophytes infection in a silver fox (Vulpes vulpes) from a breeding farm. Med Mycol 57:171-180. https:// doi.org/10.1093/mmy/myy011

56. Drouot S, Mignon B, Fratti M et al (2009) Pets as the main source of two zoonotic species of the Trichophyton mentagrophytes complex in Switzerland, Arthroderma vanbreuseghemii and Arthroderma benhamiae. Vet Dermatol 20:13-18. https://doi.org/10. 1111/j.1365-3164.2008.00691.x

57. Gnat S, Łagowski D, Nowakiewicz A, Dyląg M (2021) A global view on fungal infections in humans and animals: infections caused by dimorphic fungi and dermatophytoses. J Appl Microbiol n/a. https://doi.org/10.1111/jam.15084

58. Hsieh A, Quenan S, Riat A et al (2019) A new mutation in the SQLE gene of Trichophyton mentagrophytes associated to terbinafine resistance in a couple with disseminated tinea corporis. J Mycol Med 29:352-355. https://doi.org/10.1016/j.mycmed.2019. 100903

59. Järv H, Uhrlass S, Simkin T et al (2019) Terbinafine resistant Trichophyton mentagrophytes genotype VIII, Indian type, isolated in Finland. J Fungi 5:117-118

60. Manoyan M, Sokolov V, Gursheva A et al (2019) Sensitivity of isolated dermatophyte strains to antifungal drugs in the Russian Federation. J Fungi 5:114

61. Burmester A, Hipler UC, Hensche R et al (2019) Point mutations in the squalene epoxidase gene of Indian ITS genotype VIII T. mentagrophytes identified after DNA isolation from infected scales. Med Mycol Case Rep 26:23-24. https://doi.org/10.1016/j. mmcr.2019.09.001

62. Mukherjee PK, Leidich SD, Isham N et al (2003) Clinical Trichophyton rubrum strain exhibiting primary resistance to terbinafine. Antimicrob Agents Chemother 47:82-86. https://doi.org/10.1128/ AAC.47.1.82-86.2003

63. Singh A, Masih A, Khurana A et al (2018) High terbinafine resistance in Trichophyton interdigitale isolates in Delhi, India harbouring mutations in the squalene epoxidase gene. Mycoses 61:477-484. https://doi.org/10.1111/myc.12772

64. Rudramurthy SM, Shankarnarayan SA, Dogra S, et al (2018) Mutation in the squalene epoxidase gene of Trichophyton 
interdigitale and Trichophyton rubrum associated with allylamine resistance. Antimicrob Agents Chemother 62: https://doi.org/10. 1128/AAC.02522-17

65. Osborne CS, Leitner I, Favre B, Ryder NS (2005) Amino acid substitution in Trichophyton rubrum squalene epoxidase associated with resistance to terbinafine. Antimicrob Agents Chemother 49:2840-2844. https://doi.org/10.1128/AAC.49.7.2840-2844. 2005

66. Osborne CS, Leitner I, Hofbauer B et al (2006) Biological, biochemical, and molecular characterization of a new clinical Trichophyton rubrum isolate resistant to terbinafine. Antimicrob Agents Chemother 50:2234-2236. https://doi.org/10.1128/AAC. 01600-05

67. Araújo CR, Miranda KC, De Fernandes OFL et al (2009) In vitro susceptibility testing of dermatophytes isolated in Goiania, Brazil, against five antifungal agents by broth microdilution method. Rev Inst Med Trop Sao Paulo 51:9-12. https://doi.org/10.1590/S003646652009000100002

68. Saunte DML, Hare RK, Jørgensen KM, et al (2019) Emerging terbinafine resistance in Trichophyton: clinical characteristics, squalene epoxidase gene mutations, and a reliable EUCAST method for detection. Antimicrob Agents Chemother 63: https:// doi.org/10.1128/AAC.01126-19

69. Čmoková A, Kolařík M, Dobiáš R et al (2020) Resolving the taxonomy of emerging zoonotic pathogens in the Trichophyton benhamiae complex. Fungal Divers 104:333-387. https://doi.org/ 10.1007/s13225-020-00465-3

70. Overgaaw PAM, van Avermaete KHA, Mertens CARM et al (2017) Prevalence and zoonotic risks of Trichophyton mentagrophytes and Cheyletiella spp. in guinea pigs and rabbits in Dutch pet shops. Vet Microbiol 205:106-109. https://doi.org/10.1016/j. vetmic.2017.05.008

71. Needle DB, Gibson R, Hollingshead NA, et al (2019) Atypical dermatophytosis in 12 North American porcupines (Erethizon dorsatum) from the northeastern United States 2010-2017. Pathogens 8

72. Gnat S, Łagowski D, Nowakiewicz A, Zięba P (2018) Tinea corporis by Microsporum canis in mycological laboratory staff: unexpected results of epidemiological investigation. Mycoses 61:945-953. https://doi.org/10.1111/myc.12832

73. Tizzani P, Gallo MG, Peano A et al (2007) Dermatophytosis caused by Microsporum canis in eastern cottontail (Sylvilagus floridanus). Eur J Wildl Res 53:238-240. https://doi.org/10.1007/ s10344-007-0088-0

74. Gallo MG, Lanfranchi P, Poglayen G et al (2005) Seasonal 4-year investigation into the role of the alpine marmot (Marmota marmota) as a carrier of zoophilic dermatophytes. Med Mycol 43:373-379. https://doi.org/10.1080/13693780400008282 